\title{
Prospects of using simulations to study the photospheres of brown dwarfs
}

\author{
Hans-Günter Ludwig ${ }^{1}$ \\ ${ }^{1}$ CIFIST, GEPI, Observatoire de Paris-Meudon, 92195 Meudon, France \\ email: Hans.Ludwig@obspm.fr
}

\begin{abstract}
We discuss prospects of using multi-dimensional time-dependent simulations to study the atmospheres of brown dwarfs and extrasolar giant planets, including the processes of convection, radiation, dust formation, and rotation. We argue that reasonably realistic simulations are feasible, however, separated into two classes of local and global models. Numerical challenges are related to potentially large dynamic ranges, and the treatment of scattering of radiation in multi-D geometries.
\end{abstract}

Keywords. Hydrodynamics, convection, radiative transfer, methods: numerical, stars: atmospheres, stars: low-mass, brown dwarfs

\section{Introduction}

The increasing number of brown dwarfs and extrasolar planets of spectral class L and later discovered by infrared surveys and radial velocity searches has spawned a great deal of interest in the atmospheric physics of these objects. Their atmospheres are substantially cooler than, e.g., the solar atmosphere, allowing the formation of molecules, or even liquid and solid condensates - in astronomical parlance usually referred to as "dust". Convection is a ubiquitous phenomenon in these atmospheres shaping their thermal structure and the distribution of chemical species. Hydrodynamical simulations of solar and stellar granulation have become an increasingly powerful and handy instrument for studying the interplay between gas flows and radiation. In this paper we discuss the prospects of developing similar multi-dimensional and time-dependent simulations of very cool atmospheres. The most important additional process - in view of previous developments for hotter atmospheres - that one needs to tackle is the dust formation coupled to the hydrodynamic transport processes and radiative transfer.

In the following, we shall take a slightly broader point of view than just considering brown dwarf (hereafter BD) atmospheres and include also the atmospheres of extrasolar giant planets (hereafter EGPs) in the discussion since their atmospheric dynamics is controlled by similar processes as brown dwarfs atmospheres.

As we shall see later, computational limitations do not allow to address the problem of the atmospheric dynamics as a whole, i.e., studying the global atmospheric circulation together with convective flows taking place at relatively small spatial scales given by the the pressure scale height at the stellar surface. Consequently, modelers have addressed the problem of the local and global circulation separately. Since many BDs and EGPs are rapid rotators, rotation constitutes another important process which one needs to take into consideration for the global circulation.

We would like to emphasize that we are discussing the perspectives for multi-D timedependent simulations. Considerable insight has already been gained by the development 
of one-dimensional, hydrostatic, time independent model atmospheres (hereafter "standard" models) for L- and T-type objects (e.g., Burrows et al. 2006, Tsuji 2002, Allard et al. 2001, Ackerman \& Marley 2001), and we draw from this work. Simulations will augment our understanding of BD and EGP atmospheres by adding information about the detailed cloud meteorology on the local scale of convective cells, as well as on the scale of the global wind circulation pattern. This includes further characterization of the effects of irradiation in close-in EGPs. The simulation of the atmospheric dynamics might also add to our knowledge about local dynamo action in substellar objects, and acoustic activity contributing to the heating of chromospheres.

\section{Micro-physical input}

In order to perform realistic simulations micro-physical input data must be available radiative opacities, equation-of-state (EOS), and a kinetic model describing the formation of dust grains. The requirements are similar to those for standard models, and consequently in simulation work one can usually take recourse to the descriptions developed for 1D models - largely on the same level of sophistication. In all three before-mentioned areas substantial progress has been made over the last decade, spawned by the discovery of the first brown dwarf and EGP in 1995. In particular, since the early work of Rossow (1978) kinetic models describing the nucleation, growth, and evaporation of dust grains under conditions characteristic of brown dwarf atmospheres have been developed (see Helling et al. 2004 and references therein). Hence, the present input data allow to set-up simulations on a sufficiently realistic level.

\section{Time scales: convection, radiation, dust, rotation, \& numerics}

To obtain insight into potential challenges one faces in simulations of the dynamics of brown dwarf and EGP atmospheres it is illuminating to take a look at the characteristic time scales of the governing physical processes. Figure 1 depicts these time scales in a representative brown dwarf model atmosphere at $T_{\text {eff }}=1800 \mathrm{~K}$ and $\log g=5.0$ of solar chemical composition. The model comprises the stellar photosphere and the uppermost layers of the convective stellar envelope. Since it is expected that the cloud decks are located in vicinity of the boundary of the convective envelope (in this model located at a geometrical height of $23 \mathrm{~km}$ ) it also contains the layers in which the dust harboring layers are expected. We emphasize that the model structure is taken from an experimental hydrodynamical simulation in which dust formation was not taken into account. Since we are interested here in order of magnitude estimates only, this is not a critical issue.

In figure 1 the line labeled "C-F-L" computed as the sound crossing time over a pressure scale height depicts the upper limit of the time step which is allowed in an explicit hydrodynamical scheme due to the Courant-Friedrichs-Levy stability criterion. Depending on the actual resolution of the numerical grid this number may be one to two orders of magnitude smaller than indicated. The line labeled "convection" depicts the modulus of the Brunt-Vaiäsälä period providing a measure of the time scale on which the convective flow evolves. Two lines labeled "radiation" indicate the time scale on which radiation changes the thermal energy content of the gas. The dashed-dotted line is computed using Rosseland mean opacities which give the correct behavior in the optical thick layers, the solid line is based on Planck mean opacities which give a better representation in the optically thin layers. For the rotational period depicted by the dashed line labeled "rotation" we took a representative value close to one day. For three different dust grain diameters of 1,10 , and $100 \mu \mathrm{m}$ we plotted the sedimentation ("rain out") time scale 


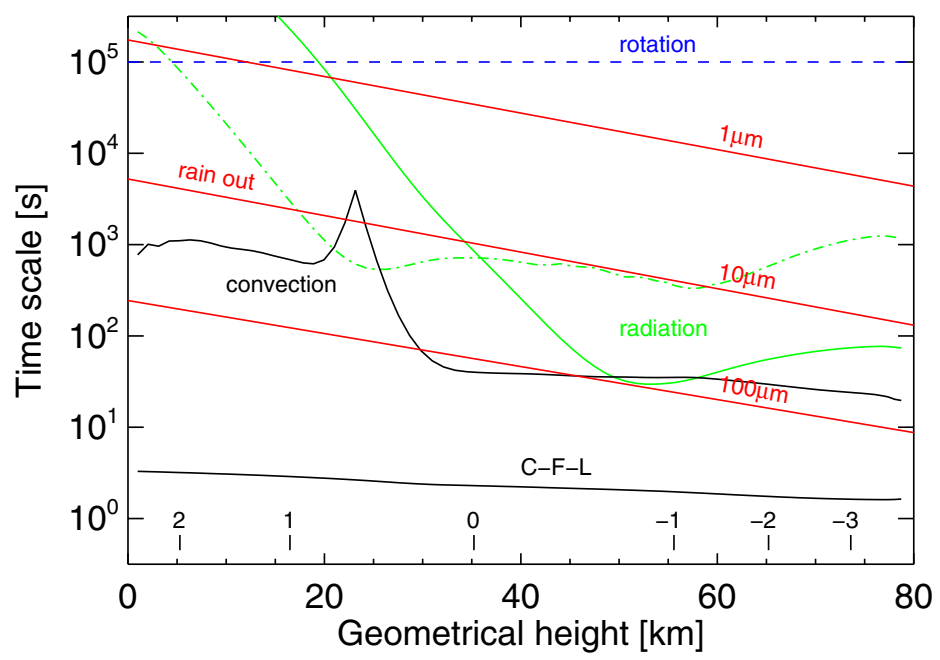

Figure 1. Characteristic time scales of various processes in a brown dwarf atmosphere of $T_{\text {eff }}=1800 \mathrm{~K}$ as a function of geometrical height. The tick marks close to the abscissa indicate the (log Rosseland) optical depth. For details see text.

taken as drifting time over a pressure scale height. The drift velocities were taken from the work of Woitke \& Helling 2003. We did not depict the formation time scale of the dust grains in the figure: for grains of $100 \mu \mathrm{m}$ diameter it is of the same order as the sedimentation time scale. Consequently, it is unlikely that larger grains can stay in brown dwarf atmospheres. The formation time scale becomes rapidly shorter for smaller grains so that they can be considered being essentially formed in quasi-static phase-equilibrium (see also Helling 2005).

Computing resources available today typically allow to simulate a dynamical range in time of $10^{4} \ldots 10^{5}$, and $10^{2} \ldots 10^{3}$ per dimension (for 3D models) in space. From figure 1 we conclude that it should be feasible to include convection, radiative transfer effects, and dust formation in a simulation of a BD/EGP atmosphere. The simultaneous inclusion of rotation is beyond reach, in particular if one takes into consideration that one would like to simulate many rotational periods to obtain a statistically relaxed state. However, the substantial difference between the time scales on which rotation and convection operate moreover indicates that rotation is dynamically not relevant for the surface granulation pattern in BD/EGPs.

A rather strong modeling limitation comes about by the large spatial scale separation between the typical size of a convective cell and the global scale of a BD or EGP of about $10^{4}$, at best reduced to $10^{3}$ for the case of young, low mass EGPs. Hence, typical BD/EGP conditions are hardly within reach with 3D models, and the steep increase of the computational cost with spatial resolution (for explicite numerical schemes with $(\Delta x)^{4}$ ) makes it likely that this situation prevails during the nearer future. We expect that 3D simulations will for some time be either tailored to simulate the global meteorology, or will be restricted to local models simulating the convective flow in detail.

Figure 2 illustrates the kinematics of the flow in a local BD simulation analogous to figure 1 . The horizontal root-mean-square of the vertical velocity component is depicted by the diamond symbols. The key-point to note is that the convective motions proper are largely confined to the convectively unstable layers. The velocities in the convectively stable layers with $\log \tau<0$ are almost exclusively related to sound waves. As essentially oscillatory motions they are ineffective for mixing so that they provide little updraft 


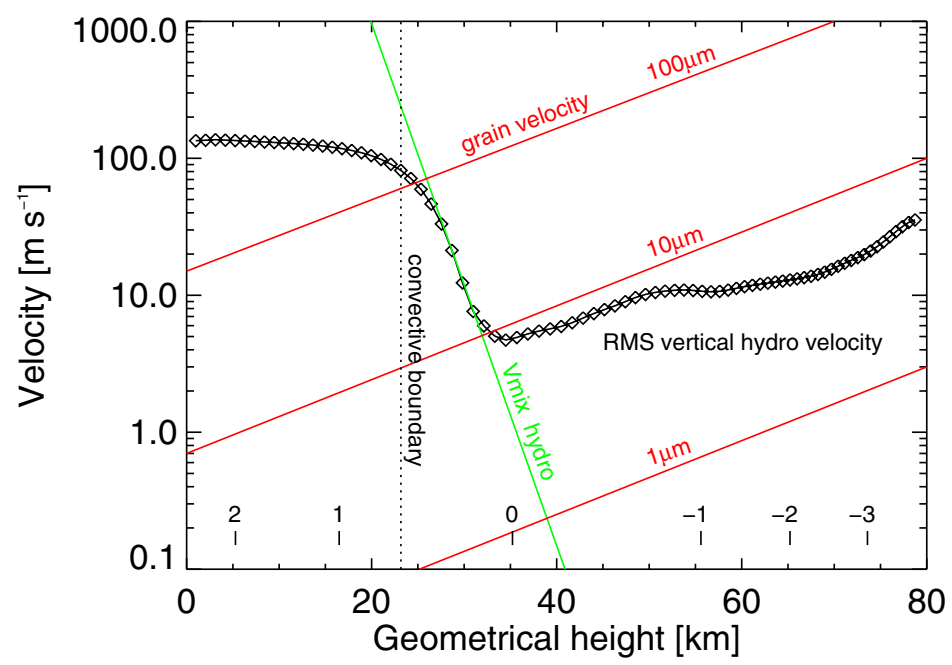

Figure 2. Characteristic velocities in brown dwarf atmosphere analogous to figure 1.

to keep dust grains aloft in the atmosphere. The green line is illustrating an estimate of the effective mixing velocity provided by the convective motions. The decline of the amplitude is rather steep - in the test model with a scale height of about $1 / 3$ of the local pressure scale height.

Comparing the mixing velocities with typical grain sedimentation velocities indicates that the kinematics could support cloud decks in the convective zone and a thin adjacent overshooting layer at its top boundary. Fitting observed spectra Burrows et al. (2006) find a preference for a rather large grain size of $\approx 100 \mu \mathrm{m}$ in $\mathrm{BD}$ atmospheres. This would make the grain sedimentation velocities comparable to convective velocities which is numerically uncritical. More demanding would be small grain sizes. The distribution of small grains would hinge on the capability of a numerical code to deal with large velocity ranges. Any non-physical diffusivity in a code can artificially extend the region over which clouds of small grains could exist.

\section{The multi-D story so far}

A number of simulations of BD/EGP atmospheres have been already conducted in $2 \mathrm{D}$ and $3 \mathrm{D}$ geometry. Here, the problem of the circulation between the day- and nightside of close-in EGPs ("hot Jupiters") achieved particular attention. However, to our knowledge none of the studies has addressed the coupled problem of hydrodynamics, dust formation, radiation, and rotation, but rather they have focused on different parts of the overall problem. We would like to refer the interested reader to Showman \& Guillot (2002), Cho et al. (2003), Burkert et al. (2005), and the follow-up work of these groups.

\section{Serendipity}

In the previous sections we summarized expectations about the insights one might gain, and challenges one might face when trying to construct multi-D models for BD/EGP atmospheres. We added figure 3 as a reminder that of course the unforeseen results are the most interesting ones. Figure 3 illustrates a slight but distinct change of the granulation pattern between the familiar solar granulation and granulation in an 


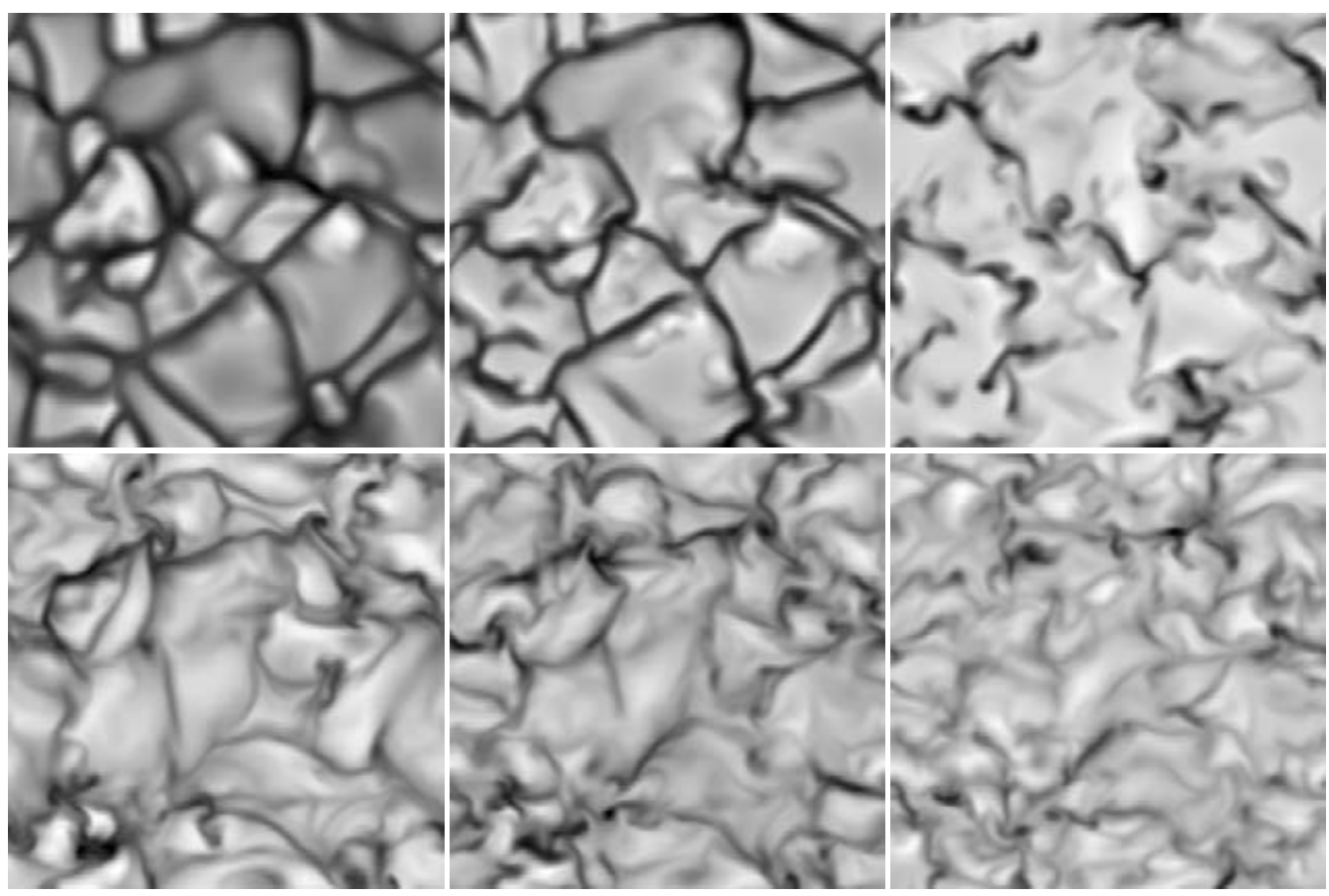

Figure 3. Grey-scale images of the vertical velocity component of a solar hydrodynamical model (top row) and an M-dwarf model (bottom row). From left to right, the velocities are depicted at (Rosseland) optical depth unity as well as one and two pressure scale heights below that level in the respective models. The absolute image scales are $5.6 \times 5.6 \mathrm{Mm}^{2}$ for the solar and $0.25 \times 0.25 \mathrm{Mm}^{2}$ for the M-dwarf model.

M-dwarf. E.g., similarly and perhaps more drastically the formation of dust in BD/EGP atmospheres might modulate the convective dynamics in unexpected ways - who knows.

\section{Conclusions}

Reasonably realistic local or global models of brown dwarf and extrasolar giant planet atmospheres coupling hydrodynamics, radiation, dust formation, and rotation are numerically in reach at present. However, "unified" models spanning all spatial scales from the global scale down to scales resolving the flow in individual convective cells are stretching the computational demands beyond normally available capacities. Hence, we expect that a separation between local and global models will prevail during the nearer future. Whether this will turn out to be a severe limitation remains to be seen. While apparently subtle we would like to point to the solar dynamo problem where the still not fully satisfactory state of affairs might be related to the lack of the inclusion of small enough scales when modeling the global dynamo action. In BD/EGP atmospheres it is perceivable that the local transport of momentum by convective and acoustic motions might alter the global flow dynamics - in the simplest case by adding turbulent viscosity.

If the sizes of dust grains in BD/EGP atmospheres turn out to be small, and the grains consequently exhibit low sedimentation speeds, numerical simulations must have the ability to accurately represent the large dynamic range between grain and convective/acoustic velocities. Overly large numerical diffusivities artificially enlarge the height range over which cloud decks can persist.

Standard model atmospheres are treating the wavelength-dependence of the radiation field commonly in great detail which is not possible in the more demanding multi-D 
geometry of simulation models. An approximate multi-group treatment of the radiative transfer has been developed for simulations of stellar atmospheres which has also been proven to provide reasonable accuracy at acceptable computational cost in cooler (Mtype) atmospheres. We expect that the scheme also works for even cooler atmospheres. However, one simplification usually made is treating scattering as true absorption. Depending on the specific dust grain properties this approximation might need to be replaced by a more accurate treatment of scattering. Hence, another challenge a modeler might face is to device a computationally economic scheme to treat scattering in the time-dependent multi-D case.

\section{References}

Ackerman, A.S. \& Marley, M.S. 2001, ApJ 556, 872

Allard, F., Hauschildt, P.H., Alexander, D.R., Tamanai, A. \& Schweitzer, A. 2001, ApJ 556, 357

Burkert, A., Lin, D.N.C., Bodenheimer, P.H., Jones, C.A. \& Yorke, H.W. 2005, ApJ 618, 512

Burrows, A., Sudarsky, D. \& Hubeny, I. 2006, ApJ 640, 1063

Cho, J.Y.-K., Menou, K., Hansen, B.M.S. \& Seager, S. 2003, ApJ 587, 117

Helling, Ch., 2005, in: Proceeding of he workshop on Interdisciplinary Aspects of Turbulence, Garching: Max-Planck-Institut für Astrophysik, eds.: F. Kupka, W. Hillebrandt, p. 152

Helling, Ch., Klein, R., Woitke, P., Nowak, U. \& Sedlmayr, E. 2004, A\&A 4 423, 657

Rossow, R.W. 1978, Icarus 36, 1

Showman, A.P. \& Guillot, T. 2002, A\&A 385, 166

Tsuji, T. 2002, ApJ 575, 264

Woitke, P. \& Helling, Ch. 2003, A\&A 399, 297

\section{Discussion}

F. KuPKA: Considering the complexity of molecular opacity I am actually surprised how robust the opacity binning seems to be.

LUDWIG: Tests have been performed for M-type stars where the effect of many millions of - primarily molecular - lines is captured quite well. As for brown dwarfs: the dust opacity has a rather smooth functional dependence on wavelength. Hence, it should be easy, but scattering is a problem.

I.W. RoxBurgh: You said overshooting was small, could you quantify this in terms of local scale height?

LUDWIG: The velocity amplitude declines exponentially with a scale height of about $1 / 3$ of the local pressure scale height. In solar models the scale height of decline is about six times larger. However, keep in mind that the hydrodynamical model presented here is experimental, in particular it does not include any effects of dust formation.

C. Helling: Your wish list implies that no progress has been made in the brown dwarf modeling. Additionally, I am convinced that we will need to work on both sides: on 1D models which are fast and applicable, not only on 3D models though they will play an important role.

LuDWIG: My wish list was intended as overall collection of things we would like to understand about brown dwarf atmospheres. Progress related to the various points has indeed already been made. Concerning the mutual role of $1 \mathrm{D}$ and 3D models, I fully agree. 3D models should address crucial aspects that are in principle not accessible in 1D. Insight emerging from 3D models should then be transferred to $1 \mathrm{D}$ models. 